\title{
Meillet et les études sur le français
}

\author{
Gabriel Bergounioux, ${ }^{1}$ \\ ${ }^{1}$ LLL (UMR 7270), Université d'Orléans, France
}

\begin{abstract}
Résumé. Antoine Meillet (1866-1936) est connu pour ses contributions à la grammaire comparée des langues indo-européennes et ses interventions sur le français pourraient paraitre secondaires. Pourtant deux types d'intervention retiennent l'attention dans la bibliographie. D'une part, la réflexion sur le statut politique des langues dans le bouleversement des revendications nationales après la Première Guerre Mondiale. D'autre part, une réflexion générale, à partir de l'exemple du français, sur les conditions sociales des transformations diachroniques - en particulier le rôle des variations internes et des contacts de langue (substrat, bilinguisme) contrecarrés par la fixation de l'écriture et la normalisation - et sur la reconfiguration des systèmes par la grammaticalisation et la simplification des flexions.
\end{abstract}

\begin{abstract}
Meillet's French language studies. Antoine Meillet (18661936) is known for his contributions to the comparative grammar of IndoEuropean languages. Thus, his research on French might seem secondary. However, two types of intervention stand out in his bibliography. On the one hand, reflection on the political status of languages in the upheaval of national claims after the First World War. On the other hand, a global view, using French as a starting point, on the social conditions of diachronic transformations - especially the role of internal variations and language contacts (substrate, bilingualism) thwarted by the fixation of writing and standardization - and on the reconfiguration of systems through grammaticalization and the simplification of inflections.
\end{abstract}

Antoine Meillet a été le maître incontesté des études de grammaire comparée des langues indo-européennes et de la linguistique générale, d'abord en France puis, suite à l'effacement de l'Allemagne après 1918, à l'échelle mondiale. Pourtant sa bibliographie, considérable fait apparaître comparativement peu de contributions consacrées à la langue française.

Après avoir justifié cette relative discrétion, on considèrera deux questions qui ont amené Meillet à s'impliquer dans ces études. D'abord, en linguistique externe, on rappellera ses conceptions en matière de politique des langues afin de montrer combien l'histoire du français telle qu'il l'envisageait est fondatrice du jugement d'ensemble qu'il porte sur les transformations qui sont survenues en Europe entre les années 1890 et les années 1930. Ensuite, en linguistique interne, on examinera comment l'examen de l'évolution du français l'a conduit à une révision des conditions qui déterminent les changements de structure jusqu'à envisager l'hypothèse d'un « développement des langues » assez semblable au «progrès » dont avait parlé Bréal avant lui. On n'a pas inclus dans la recension les nombreuses références qui, au fil des pages, servent d'illustration à la perspective diachronique qui est la sienne. Ne seront pas traitées non plus ses interventions ponctuelles sur des questions d'enseignement et de norme. Elles concernent moins l'élaboration de la théorie linguistique proprement dite que les conclusions qui pouvaient en être tirées à l'intention du grand public. A l'occasion de polémiques journalistiques qu'il faudrait contextualiser, le savant entreprenait de réagir aux propos de publicistes à l'intention de lecteurs qui, parce qu'ils ne disposaient pas de toute l'information requise, devaient être éclairés sur la réalité des enjeux.

\section{Un partage des études}

\footnotetext{
${ }^{1}$ Corresponding author : gabriel.bergounioux@univ-orleans.fr
} 


\subsection{Comparatisme roman, comparatisme indo-européen}

Dès le $\mathrm{XVI}^{\mathrm{e}}$ siècle, les études philologiques ont ménagé une place éminente à la langue française pour des raisons politiques et pédagogiques. Cette prééminence, dont on fait remonter l'officialisation à l'ordonnance de Villers-Cotterêts, avait été sanctionnée en 1635 par la création de l'Académie française qui confiait l'autorité sur la langue aux écrivains. L'irruption de la grammaire historique au XIX ${ }^{\mathrm{e}}$ siècle et le transfert depuis l'Allemagne des enseignements comparatistes ont abouti à un dédoublement de la compétence entre les auteurs et les professionnels de la parole publique (orateurs politiques, journalistes) et les professeurs nommés dans l'enseignement supérieur à partir des années 1850-1860.

Voici une représentation schématique de la répartition des formations autour de 1890 en dehors des langues non indo-européennes et des enseignements pratiques - recensées à partir des intitulés (Carle 1985 ; Bergounioux 1990) :

Français, latin et langues romanes

Facultés des lettres ( 30 chaires)

Ecole des Chartes ( 2 chaires)

E.P.H.E. (2 directions d'étude)

Collège de France ( 6 chaires)
Grammaire comparée (indo-européen)

Paris et Lyon ( 2 chaires)

Ecole Langues Orientales ( 2 chaires)

E.P.H.E. (4 directions d'étude)

Collège de France ( 7 chaires)

Tab. 1 Chaires et directions d'étude consacrées aux langues indo-européennes ( ca 1890)

L'implantation du français et du latin dans les facultés tient pour une large part à une association de l'enseignement de la langue avec la littérature. Intégrées au cursus classique, en continuité avec les cours dispensés en lycée, ces deux disciplines - et à un moindre degré le grec - attirent l'essentiel d'effectifs qui demeurent modestes : en 1885-1890 (Prost 1968), il y avait 2400 étudiants dans les facultés de lettres où étaient délivrées annuellement 400 licences. Inversement, l'un des problèmes récurrents du comparatisme en France tient à la difficulté de la transmission du fait d'un très faible auditoire et de l'absence de carrières correspondant au diplôme.

A l'initiative des gouvernements, notamment des hauts fonctionnaires qui impulsaient les réformes contre la volonté d'un corps enseignant conservateur (les nominations supposaient d'avoir prêté serment à Napoléon III), les facultés de lettres avaient été contraintes par la redéfinition des épreuves de l'agrégation d'introduire des éléments de grammaire historique dans les leçons sur l'ancien français et d'étudier les œuvres de la littérature médiévale. Faute de personnels qualifiés, ces cours avaient d'abord été confiés à des maîtres de conférences, un nouveau corps créé dans l'enseignement supérieur au milieu des années 1870. Nombre de ces jeunes titulaires ont ensuite été promus professeurs : c'est le cas d'A. Darmesteter à Paris, de L. Clédat à Lyon, de C. Chabaneau à Montpellier, d'E. Bourciez à Bordeaux... Des tentatives homologues pour une redéfinition des études latines ou grecques n'ont pas abouti à des résultats probants en dépit des efforts de Louis Havet en latin; quant aux cours de sanscrit, assurés à Paris par A. Bergaigne et à Lyon par P. Regnaud, institués en dehors des cursus obligatoires, ils n'ont jamais eu qu'un succès d'estime.

La nécessité de porter l'enseignement supérieur français à un niveau comparable à celui atteint par les universités allemandes qui, depuis Humboldt, avaient su associer recherche et formation, a été exacerbée par les conséquences de la guerre de 1870. Inspiré par un jugement dont les grandes lignes sont esquissées par Renan (1871), imputant la défaite au retard en matière d'éducation, le gouvernement s'est engagé dans une série de remaniements qui, de 1868 à 1878, ont reconfiguré les filières post-bac dans un cadre qui ne sera définitivement bouleversé qu'en 1968 .

Dès sa mise en place, l'organisation des enseignements linguistiques a établi séparément la grammaire comparée des langues indo-européennes et l'étude du français et des langues issues du latin. En même temps que des subventions étaient accordées pour des bourses d'études outre-Rhin et le financement des traductions et de l'édition des grammaires de F. Bopp par M. Bréal (1866-1875) et de F. Diez par G. Paris (1874-1876), la création de la IV section de l'EPHE entérinait, dans ses statuts de fondation, la séparation entre ce qui concernait le français et les langues romanes d'un côté, attribués à G. Paris, et toutes les autres langues indo-européennes de l'autre, latin compris, avec une direction d'études distincte confiée à M. Bréla.

Ainsi Meillet, plus jeune d'une génération (Bréal est né en 1832, Paris en 1839 et Meillet en 1866) est fort peu intervenu dans les études sur le français qui restaient l'apanage de Gaston Paris et Mario Roques à l'EPHE et au Collège de France où la littérature et la philologie dominaient, et plus encore à la Sorbonne avec d'un côté les chaires d'Antoine 
Thomas en " philologie française » et de l'autre Ferdinand Brunot en « histoire de la langue française ». C'est seulement sur des points de rencontre comme le statut politique des langues et la dialectologie, la recherche en étymologie et le changement linguistique que se sont produites les rencontres.

\subsection{Les interventions de Meillet sur le français}

Si on n'a pas intégré dans la recension des interventions que Meillet a faites ceux des deux mille comptes rendus qui pouvaient concerner le français, on relèvera qu'aucun n'a été publié dans l'un des périodiques animés par les romanistes bien que Maurice Grammont, qui assistait avec Meillet aux cours de Saussure, ait dirigé la Revue des Langues Romanes à Montpellier et qu'il se soit à quatre reprises manifesté dans des périodiques relevant de ce domaine (Revue de Philologie Française, Romanic Review, Revue de Linguistique Romane). Aucun livre, sur les vingt-trois parus du vivant de l'auteur, ne traite du français alors qu'on y trouve des grammaires sur l'arménien, le polonais, le serbo-croate, des études sur les langues germaniques, le grec, le latin, les langues slaves. Sur les 540 articles répertoriés par Benveniste (bibliographie reproduite avec les corrections de P. Swiggers dans Bergounioux \& Lamberterie 2006), dix-huit seulement font référence au français :

[120] (1909). Sur la disparition des formes simples du prétérit. GermanischRomanisch Monatschrift, 521-526.

[168] (1913). La crise de la langue française. Revue Bleue, 385-389.

[290] (1920). La linguistique et l'enseignement de la grammaire. Bulletin de la Société Française de Pédagogie, 93-98.

[291] (1919). De la formation des maîtres primaires. Revue de Métaphysique et de Morale XXVI, 765-769.

[291bis] (1920). L'enseignement supérieur et la formation des maîtres. Les Semailles, bulletin officiel du syndicat des membres de l'enseignement de la Seine, II, mars, 1112.

[293] (1920). Le Bulletin de guerre de l'Alliance Française. Bulletin de l'Alliance Française, 12-16.

[315] (1921). Quelques remarques sur des mots français : I. Pourboire II. J'ai bu III. De la langue qu'on parle aux enfants IV. Jardinière d'enfants. Bulletin de la Société de Linguistique de Paris XXI, 166-168.

[405] (1926). Sur la valeur du mot français jument. Archivio Glottologico Italiano, 20, 147-150 - LHLG 2.

[406] (1926). De l'état actuel de la langue française. Bulletin de la Société Française de Pédagogie, 674-684.

[412] (1927). Réponse à une observation (sur fr. lapin). Bulletin de la Société de Linguistique de Paris XXVII, 122-123.

[428] (1927). Aujourd'hui. Mélanges de philologie et d'histoire offerts à Antoine Thomas par ses élèves et ses amis, 291-293 - LHLG 2.

[429] (1927). La nécessité de recueillir le vocabulaire des parlers locaux. Société d'Emulation du Bourbonnais, 9-11.

[444] (1928). Lettre [sur la nécessité de constituer un vocabulaire historique complet de la langue française]. Le Temps, 31 mars.

[445] (1928). Lettre [sur la prosodie du mot fr. tiède]. Revue de Philologie Française, $40,62-64$.

[478] (1930). La notion de radical en français. The Romanic Review XXI, 291-295 LHLG 2.

[492] (1931). A propos de "il est vache" et de "j'ai très faim". Revue de Philologie Française, 43, 47-49 - LHLG 2.

[506] (1933). Lettre [sur la prononciation française du latin]. L'Europe nouvelle, 7 janvier, 17.

[507] (1932). Préface. Dictionnaire étymologique de la langue française d'O. Bloch et W. von Wartburg, V-XV, 1932 - LHLG 2.

LHLG = Linguistique historique et linguistique générale (recueil d'articles, 2 volumes)

BSL $=$ Bulletin de la Société de Linguistique de Paris

Tab. 2 Articles de Meillet consacrés au français. Le chiffre entre crochets correspond au numéro de l'item dans la bibliographie ; il est parfois distinct de l'ordre chronologique. La mention $L H L G 2$ signale une reprise dans Meillet (1936).

Peuvent être ajoutés deux articles à visée panromane : 
[349] (1922). L'unité romane. Scientia, XXXI, 149-153.

[394] (1925). Les langues romanes et les tendances des langues indo-européennes.

Revue de Linguistique Romane I, 1-8 - LHLG 2.

Tab. 3 Articles de Meillet consacrés aux langues romanes

On note que l'index des langues qu'a dressé Benveniste à la fin de sa bibliographie ne comprend pas d'entrée pour le français.

\section{Le statut politique des langues : le cas du français}

\subsection{La fabrique du français littéraire}

Dans Les langues dans l'Europe nouvelle (1918, cité d'après la deuxième édition de 1928 revue et complétée d'une statistique des langues par L. Tesnière), désormais notée (Meillet 1928b), Meillet consacre un chapitre entier au « Français littéraire ». Il en suit l'élaboration à la Renaissance, au moment où le mouvement des idées et la révolution de la diffusion par l'imprimerie contraignent les érudits à forger une terminologie et un style qui soient vernaculaires tout en empruntant au latin l'essentiel des ressources requises par l'entreprise. Ce recours massif à un état ancien - le français est une évolution régionale du latin - a opéré une séparation entre l'écrit et les pratiques concrètes, orales, des locuteurs. Plus abstraites, donc plus complexes, les représentations conceptuelles et l'expression sont devenues encore plus élitistes avec la greffe de termes grecs qui n'avaient pas eu de représentants en moyen français.

Meillet insiste sur une normalisation de la langue qui a été géographique - « Avec Malherbe et le XVII ${ }^{\mathrm{e}}$ siècle on revient au pur français de Paris » (Meillet 1928b, 169) - et sociale. Les remarqueurs, les grammairiens et les critiques avaient un statut social qui les plaçaient à distance de la cour et du peuple, mais aussi des écoles liées à l'Eglise et au latin. Une filiation revendiquée des capitales culturelles, d'Athènes à Rome et de Rome à Paris, aboutit au constat d'une variation sociale qui se reproduit à des siècles de distance :

La situation du français est, depuis la première moitié du XVII ${ }^{\mathrm{e}}$ siècle, celle du grec depuis le III $^{\mathrm{e}}$ siècle av. J.-C. et du latin durant la période impériale : une langue écrite arrêtée qui se défend contre le changement, mais qui n'évite pas certains fléchissements ; une langue parlée, qui se rapproche plus ou moins de la langue écrite, suivant le degré de culture de ceux qui parlent, et qui présente toutes les nuances possibles depuis le patois à peine francisé du paysan ou le parler faubourien de certains ouvriers grossiers jusqu'au parler des cercles les plus cultivés et les plus distingués, presque identique à la langue écrite. (Meillet 1928b, 171)

$\mathrm{Ne}$ voyant dans les proclamations du romantisme que l'abandon d'un vocabulaire poétique suranné, Meillet assigne l'irruption du vocabulaire populaire dans la langue littéraire aux œuvres des écrivains des tranchées (René Benjamin, Henri Barbusse). Après que ces témoignages ont été qualifiés esthétiquement, la langue écrite ne coïncide plus désormais avec tous les usages soutenus, préfigurant une exténuation d'emplois poétiques de plus en plus coupés des usages quotidiens.

La langue commune française est donc une langue traditionnelle, littéraire, aristocratique, et qui ne peut être maniée d'une manière courante que par des personnes ayant un degré très élevé de culture. Elle a été créée par le travail d'une élite intellectuelle et d'une élite sociale. C'est une sorte d'idéal dont les Français se rapprochent plus ou moins sans qu'aucun arrive à le réaliser. (Meillet 1928b, 174)

Meillet campe sur cette position lorsqu'il est amené à prendre parti sur la « crise du français » qu'il perçoit comme un effet de cet écart. Il souligne que la surreprésentation des journalistes et des avocats dans la représentation politique, c'est-à-dire de professionnels d'une langue écrite, académique, constitue une menace pour la représentativité populaire qui exacerbe la contradiction entre l'affirmation constitutionnelle des principes démocratique et la réalité de l'exercice du pouvoir. Au demeurant, cette situation est considérée comme représentative du statut des principales langues de l'Europe occidentale :

A l'Ouest, des aristocraties et des bourgeoisies bien organisées depuis longtemps se sont donné des langues communes fondées sur les parlers des villes du pays : anglais, allemand, français, italien, espagnol, portugais. La puissance de ces grandes langues est telle qu'elles refoulent d'un mouvement ininterrompu les parlers locaux. Les parlers ruraux imitent de plus en plus les parlers urbains, au point de perdre tous leurs caractères propres. (Meillet 1928b, 176) 
La vision demeure métropolitaine, sans mention des effets de l'impérialisme. Attentif à la terminologie, Meillet distingue géographiquement parler, langue et dialecte. Si, dans son classement, il réserve la portion congrue à la différenciation sociale, il s'affranchit des considérations diachroniques pour ne conserver que trois critères : la diffusion spatiale, les prescriptions métalinguistiques et la tradition littéraire.

On entend par parler l'ensemble des moyens linguistiques employés par un groupe local à l'intérieur d'un groupe occupant une aire étendue. (...) Les parlers locaux sont souvent nommés patois. Dans la mesure où le mot langue s'oppose au mot parler, il désigne un ensemble de procédés définis, et dont on a conscience, en somme. (...) $\mathrm{Au}$ contraire, le français est une langue commune qui a des règles rigoureusement fixées, une tradition littéraire. Le français s'oppose ainsi aux parlers français. Ainsi entendu, le mot langue a un sens très élastique. La pratique comporte un flottement suivant les classes sociales, suivant les conditions d'emploi (langue parlée et langue écrite), etc. A l'intérieur d'un groupe linguistique étendu, on constate, en général, que certains parlers offrent des traits communs et que les sujets parlants de certaines régions ont le sentiment d'appartenir à un même sous-groupe : en pareil cas, on dit que ces parlers font partie d'un même dialecte. (...) on ne peut le plus souvent assigner de limite précise au dialecte, parce que chacune des particularités par lesquelles se caractérisent les dialectes, a en général une limite propre, différente de celle de toute autre particularité. ( $L H L G 2,1932,67-68$ - on a précisé la date de première publication du texte)

Partisan de regroupements continentaux autour d'un petit nombre de langues de culture (ainsi, contre Tesnière, il prône l'adoption du serbo-croate comme langue de la Slovénie), Meillet en extrapole les modalités d'imposition à partir de la situation qui a prévalu en France. A la violence symbolique inhérente au processus de substitution d'une langue à des dialectes ou à d'autres langues, il oppose le volontarisme de la Révolution qui a associé la francisation à la conquête de la liberté et de la démocratie :

Depuis la Révolution, il y a partout un désir profond de posséder la langue nationale. Tout homme ayant une situation hors de son village, toute personne même à demi cultivée ou bien passe entièrement à la langue commune ou au moins joint une connaissance entière de la langue commune à celle de son parler local. Il n'y a donc pas de question de langue. Un savant linguiste de l'Europe orientale parlait un jour de l'oppression du breton en France. L'idée ne peut que faire sourire un Français, surtout s'il est breton : par rapport au français, le breton est un outil si grossier, si peu utile qu'aucun Breton sensé ne peut songer à l'employer de préférence. Autant dire que la lampe électrique opprime la chandelle ou le bâton de résine ; autant se plaindre de ce que la moissonneuse fait du tort à la faucille. C'est si on lui imposait l'école en breton, le journal en breton qu'un paysan breton se sentirait opprimé. (Meillet 1928b, 178179)

Meillet est originaire du centre de la France, de Châteaumeillant, dans la zone de langue d'oïl qui est à la fois la plus proche du français central et de l'occitan (zone du Croissant) et il adhère au principe d'une politique de francisation par l'instruction publique dont il considère que le modèle serait transposable partout en Europe, qui ne serait pas celle d'un transfert de population comme le sont la germanisation ou la russification des territoires, voire l'anglicisation de l'Amérique du nord, de l'Océanie et de l'Afrique du Sud.

\subsection{La francisation du pays et le rôle des intellectuels}

Il y a parallèlement une revendication de préemption en faveur des intellectuels pour une définition de la forme de reconnaissance de la langue :

Parmi ces parlers [issus du latin], certains, employés par des hommes de culture supérieure, et doués de prestige, ont été imités, et il s'est constitué des langues centrales, italien, espagnol, provençal, français, qui, servant de modèles, ont influencé les parlers locaux, et qui ont fourni des instruments à des littératures nouvelles. (LHLG $2,1929,79)$

Sont discutés dans (Meillet 1928b) le cas du mouvement provençaliste, « tout littéraire » ainsi que la distinction des langues d'oïl et d'oc qu'avait refusée une génération de romanistes (G. Paris, P. Meyer) et que confirme à l'inverse Meillet tout en ne laissant aucune espérance aux régionalistes quant à une reconnaissance officielle. Concernant l'Alsace reconquise, il plaide en faveur d'un retour au statu quo ante 1870 : tolérance pour le dialecte alémanique dans la vie quotidienne mais imposition du français comme langue d'acculturation par l'école et les cadres administratifs. Au sortir de la guerre, c'était une prise de position progressiste. Parallèlement, l'évolution de la situation en Belgique est appréhendée comme une revanche flamande qui aboutit à une restriction de l'ouverture au monde alors que la Suisse est donnée 
comme le modèle d'une coexistence pacifique entre trois grandes langues de culture.

Meillet constate les effets opposés d'une francisation qui, géographiquement, facilite une large diffusion culturelle et, en retour, tend à une certaine forme d'universalisme qui s'émancipe des restrictions territoriales, mais qui, socialement, hiérarchise, voire exclut les classes populaires - en particulier rurales - des emplois d'Etat par l'imposition d'un niveau de langue, d'un style et d'une orthographe élitistes tant la relation entre la langue nationale et les parlers de France est dissymétrique :

Mais, dans la plupart des cas, c'est la langue des classes dominantes qui prévaut : le français qui se répand aujourd'hui en France n'est pas la langue du peuple ; c'est le parler de la classe cultivée du XVII' siècle à Paris. Et le travail de Gilliéron a montré que ce n'est pas le français qui se nourrit de patois ; c'est le patois qui se nourrit de français cultivé. ( $L H L G$ 2, 1931, 93)

Dans la conviction inspirée par la situation française qu'une transposition d'une diffusion linguistique qui s'est réalisée avec l'assentiment des locuteurs, sans qu'il y ait de censure à l'encontre des usages privés, est réalisable sans conflit majeur, Meillet se réfère à l'exemple de la romanisation où c'est à partir des villes, des pratiques des « bourgeois » dans l'acception étymologique, que s'est répandue et imposée la langue officielle.

L'histoire de ces créations de langues communes est diverse et souvent très compliquée. Le type le plus simple est celui du français : le parler d'une région centrale, qui est celui des chefs du pays et où la civilisation a son centre, devient intégralement la langue commune ; Paris, résidence principale du roi de France, centre naturel de la France du Nord, siège d'une Université puissante qui a eu au moyen âge une forte influence, a donné son parler à la royauté française ; dès le moyen âge, le français commun est la langue de Paris ; les textes écrits en d'autres dialectes n'ont qu'une importance secondaire et, de bonne heure, le français écrit n'est rien que la langue de Paris, telle qu'elle se fixe sous toutes sortes d'influences savantes et littéraires et telle que l'adopte l'administration royale. (LHLG 1, 1911, 123)

Il s'ensuit une inquiétude sur le morcellement en cours. L'éclatement des empires allemand, austro-hongrois, russe et turc se traduit par l'avènement d'un grand nombre de langues officielles après les traités de paix de la Première Guerre Mondiale, imposant des parlers de paysans recueillis pieusement sur les côtes baltes, dans les Carpates ou en Slovénie, en lieu et place des langues à grande diffusion que sont le russe et l'allemand. Il y aurait un affaiblissement des capacités d'expression dans les lacunes inhérentes à des parlers sans tradition littéraire, et le risque de marginalisation de population qui ne participeraient plus aux courants d'échange mondiaux.

\subsection{Le français et la civilisation européenne}

A cette fragmentation, Meillet oppose l'effort des nations romanes pour conserver, en plus du fonds commun, une identité du lexique des classes cultivées, en quoi elles diffèrent des langues slaves dont le vocabulaire quotidien est plus proche et les terminologies savantes plus éloignées. Dans l'article "Sur le sens linguistique de l'unité latine », paru opportunément dans la Revue des Nations latines en 1916 après l'entrée en guerre aux côtés de la France et de la Belgique (1914) de l'Italie (1915), du Portugal et de la Roumanie (1916), il conclut cependant : " l'unité romane actuelle tient avant tout à l'unité profonde de la civilisation européenne occidentale ». Il anticipait l'hypothèse de Whorf sur le Standard Average European mais surtout, il marquait ses distances avec les jugements chauvins qui opposent traditionnellement les Welches aux Teutons.

En 1925, chargé en tant que membre de l'Académie des Inscriptions et Belles-Lettres de prononcer l'une des conférences annuelles de l'Institut, il déclare :

Nous devons à l'aristocratie indo-européenne la base de notre organisation sociale qui a pris à Rome une forme nouvelle ; nous devons à la Grèce le système de notre pensée, et à Rome l'adaptation de cette pensée à l'usage commun; nous devons à la religion juive et à la religion chrétienne nos conceptions religieuses; nous devons à la science expérimentale des derniers siècles nos idées sur le monde. Toute l'Europe actuelle, et l'Europe de langue germanique ou slave autant que l'Europe de langue romane, a hérité de ce fonds universel, et, directement ou sous un déguisement, tous les vocabulaires intellectuels de l'Europe sont faits des mêmes éléments. Pour ce qui exprime la civilisation, il y a dans nos langues, en dépit des amours-propres nationaux, beaucoup de bien commun, presque pas de bien particulier. (LHLG 2, 1925, 42-43)

Dépassant le cadre étroit d'une observation langue par langue, Meillet commence par une appréciation sociologique de la diffusion des langues indo-européennes (une « aristocratie ») qui n'a pas malgré tout la signification que lui donnera l'avènement du nazisme, avant de renouer avec la filiation gréco-romaine et judéo-chrétienne pour dessiner l'histoire d'une 
pensée triomphante que la science prolonge. Pour l'avenir, il en appelle à un projet européen, dans l'espérance d'un rapprochement pacifique au nom de la «civilisation » (vs la « culture »).

En 1932, il reprend cette idée en ajoutant aux sciences les techniques en s'inspirant du programme de la revue Wörter und Sachen :

Il faudrait, enfin, un relevé de tous les termes de la civilisation européenne, avec les formes particulières prises dans chaque langue, car cette civilisation est une, et au fond elle a un vocabulaire un : le travail n'est pas commencé. L'extension du moulin à eau que n'a pas connu l'antiquité a donné au groupe de molinus une importance qu'on n'aperçoit que si, en même temps qu'à français moulin et meunier, on pense à Mühle et Muller de l'allemand, à mill de l'anglais. (LHLG 2, 1932b, 151)

Au-delà de l'analyse linguistique s'esquisse la vision politique d'un rapprochement entre les peuples d'Europe qui s'effectuerait par le haut, grâce à ceux qui produisent et qui transmettent les connaissances. A sa façon, Meillet s'était engagé dans cette voie, cherchant à en donner le modèle dans sa discipline en accordant son patronage au premier Congrès International des Linguistes (cat il s'agit « des linguistes » et non de « la linguistique ») tenu en territoire neutre, à La Haye, en 1928.

En s'élevant au-dessus d'une étude des langues effectuée séparément pour les rapporter à leur origine commune, le français devient l'une des composantes d'un ensemble roman dont la consistance paraît secondaire en regard de la proximité contemporaine résultant de la convergence de la civilisation européenne. Ce faisant, Meillet réexamine les circonstances du changement diachronique et ses répercussions sur la théorie comparatiste. En particulier, il est amené à critiquer la théorie d'une évolution interne des langues qui se réaliserait indépendamment de tout contact et de toute influence extérieure (l'effet des lois aveugles de la phonétique et du rôle de l'analogie chez les Junggrammatiker), qui exclurait tout critère social dans l'explication des transformations. Ce faisant, il revient à une téléologie de l'évolution, à un « progrès des langues » qu'avait défendu Bréal (1897).

\section{La transformation des langues : le français en exemple}

Le comparatisme s'est trouvé associé au mouvement de la résistance nationale de l'Allemagne contre Napoléon (François 2017). L'exactitude philologique qu'il imposait n'était détachée ni d'une relecture romantique de l'histoire de l'humanité comme le montre la réception des études indiennes par Schlegel (1837), ni de la volonté de rédimer les cultures populaires (les contes des frères Grimm). Au-delà des nombreux exemples puisés dans le passé de la langue française, que ce soit pour la commodité du lecteur ou la facilité de l'explication, Meillet s'inspirait des enseignements de la perspective diachronique pour en tirer des conclusions qui s'appliqueraient à plusieurs, voire toutes les langues.

\subsection{Les conditions d'observation en temps réel}

Le point focal des critiques de Meillet porte sur une discussion récurrente entre les linguistes et les philologues. Pour la résumer en une phrase : en quoi les documents écrits dont dispose le chercheur offrent-ils une image véritablement représentative de la langue ? Après avoir mis l'accent sur l'écart entre les usages quotidiens des locuteurs et le niveau de langue imposé dans les restitutions écrites, il donne pour exemple l'écart de compréhension des textes à deux siècles et demi de distance seulement :

(...) depuis le XVII siècle, la prononciation et la grammaire du français n'ont changé que dans le détail ; il ne faudrait pas un grand effort à un contemporain de Molière pour s'adapter au système linguistique du français d'aujourd'hui ; mais il comprendrait très mal ce qu'on lui dirait parce que les mots et le sens des mots lui seraient en grande partie nouveaux. Et quiconque n'a pas fait de la langue du XVII ${ }^{\mathrm{e}}$ siècle une étude approfondie est exposé tantôt à ne pas comprendre un texte de cette époque et tantôt à faire en le lisant de grossiers contresens, simplement parce que les mots employés sont, pour une large part, autres que les nôtres, ou qu'ils ont un sens autre. (LHLG I, 1913, 29)

Même l'observation en temps réel n'apparaît pas suffisamment convaincante, qu'il s'agisse du travail de Rousselot en phonétique expérimentale au sein de sa famille (1891) ou l'enquête de terrain menée par Gauchat (1905). L'un et l'autre demeurent limités dans leurs analyses par la description de phénomènes qui ne révèlent pas ce que sont les conditions sous-jacentes, la structure de la langue et les rapports sociaux.

Lorsque Meillet affiche son ralliement à l'école durkheimienne en publiant dans L'Année sociologique «Comment les mots changent de sens », l'année même où il s'apprête à prendre 
la succession de Michel Bréal au Collège de France, il privilégie une explication par la transmission entre les générations. Ce faisant, il réintroduit les locuteurs comme les agents d'un processus que les Junggrammatiker assignaient à des lois mécaniques :

Cette discontinuité de la transmission du langage ne suffirait à elle seule à rien expliquer, mais, sans elle, toutes les causes de changement auraient sans doute été impuissantes à transformer le sens des mots aussi radicalement qu'il l'a été dans un grand nombre de cas. (LHLG 1, 1906c, 236)

Pourtant, dans sa leçon d'ouverture, il nuance ce jugement et fait référence aux contacts en complément des théories comparatistes classiques, ajoutant aux différences d'âge la façon dont se réalise la diffusion géographique d'une innovation :

Quand ils se proposent d'expliquer les changements qui surviennent dans les langues, les linguistes recourent d'ordinaire à un très petit nombre de notions fondamentales distinctes. Ils constatent que, à certains moments, en certains lieux, la prononciation subit telle ou telle modification, et ils formulent en une loi phonétique cette modification qui atteint la prononciation en tant que telle, et indépendamment de toute considération de sens ou de rôle grammatical. (...) D'autres changements ont lieu en fonction du sens, du rôle grammatical ou syntaxique, et, bien qu'ils puissent à l'occasion avoir des résultats identiques aux précédents, ces changements dits analogiques en diffèrent par leur nature d'une manière essentielle. (...) Mais, et ceci est un troisième type distinct des deux précédents, la langue d'une localité donnée est toujours plus ou moins accessible à l'influence des populations avec lesquelles ses membres sont en rapport : on emprunte des mots, des tours syntaxiques, des formes grammaticales, des manières de prononcer à d'autres langues, à d'autres parlers ou même à des textes écrits. ( $L H L G 1,1906 \mathrm{~b}, 3-4)$

Comme exemple, Meillet donne la modification de /we/ en /wa/, e.g. "bois" /bwe/ >

$/ b w a /$, qui a été spontanée à Paris mais s'est progressivement imposée au reste du pays comme un emprunt à l'emploi apparu en Ile-de-France. La conclusion est généralisée en partant des résultats de Gilliéron et Mongin (1905) et de l'Atlas Linguistique de la France en instance de publication, et des préceptes de l'école anthropologique allemande des Wörter und Sachen:

Quand on constate l'existence d'un mot en latin et de son représentant phonétiquement correct dans un parler français moderne, on est au premier abord tenté de croire que ce mot s'est simplement transmis de génération en génération ; la géographie linguistique, combinée avec l'examen des choses et l'histoire des choses, a montré que cette vue simple était une vue inexacte ; elle a révélé des séries d'emprunts dans des cas où l'on supposait, assez naïvement, la persistance d'une même vocable durant des suites illimitées de siècles. ( $L H L G 1,1906 \mathrm{~b}, 6)$

\subsection{Le bilinguisme}

Même si elle n'a jamais été absente, la réflexion sur le rôle du bilinguisme dans le changement linguistique intervient relativement tard dans la réflexion de Meillet. A propos des langues parlées :

Sur le domaine français, il y a eu deux périodes prolongées de bilinguisme. La première est celle qui a suivi l'introduction du latin sur un territoire de langue gauloise à partir de la conquête romaine. (...) La seconde est celle qui a suivi les invasions germaniques ; du $\mathrm{VI}^{\mathrm{e}}$ au $\mathrm{IX}^{\mathrm{e}}$ siècle, la puissance politique a été aux mains des conquérants de langue germanique. (...) La coexistence de deux groupes importants, l'un par sa puissance, l'autre par sa civilisation, a eu pour conséquence nécessaire que la plupart des hommes des classes dominantes et nombre de leurs sujets ont dû pratiquer à la fois les deux langues, qui toutes les deux avaient du prestige, cas assez rare. (LHLG 2, 1931a, 92)

La substitution du latin aux langues présentes avant la conquête romaine est historiquement documentée bien que les langues condamnées, en particulier le gaulois, ne soient pas aussi bien connues. Meillet insiste avant tout sur la diffraction sociale intervenue au moment du changement de langue, un processus observable en conditions réelles dans le contexte contemporain avec pour référence de ces études les travaux de H. Schuchardt (2011)

Les faits qu'on vient de grouper font apparaître un type de complexités dont il serait juste de tenir compte. Ils montrent de quel intérêt il serait d'examiner toutes les populations bilingues, en France notamment les provinces où il s'emploie deux langues, et surtout où l'une des deux langues n'est pas défendue par une langue voisine, comme c'est le cas pour le breton et pour le basque. Des recherches minutieuses sur place devraient être entreprises. Il ne serait pas moins intéressant 
d'envisager l'arabe et le berbère par exemple en Algérie et au Maroc. (LHLG 2, 1931a, 98)

Deux exemples sont donnés afin d'illustrer les effets de substrat (du gaulois sur le latin) et de superstrat (des langues germaniques sur le proto-roman et l'ancien français). Pour l'influence du gaulois, sont mentionnés l'apparition d'une troisième série vocalique avec l'insertion $\mathrm{du} / \mathrm{y} /$ entre /i/ et /u/, l'opposition d'aperture entre les voyelles moyennes, l'amuïssement des voyelles finales et des consonnes intervocaliques avec en épilogue : «Le développement du français a donc suivi la même direction que celui des langues celtiques. » (LHLG 2, 1932, 110).

Le rôle des langues germaniques était plus délicat à évaluer, enjeu de revendications contradictoires à travers tout le $\mathrm{XIX}^{\mathrm{e}}$ siècle. Pour des raisons politiques, la romanistique allemande avait cherché à en surévaluer l'importance et la romanistique française à la sousestimer. Chez Meillet, les quelques phénomènes qu'il impute aux conséquences des grandes invasions ne sont pas présentés à l'avantage des conquérants ; ils auraient retardé une évolution où, fidèle au moins en cela à Bréal, Meillet voyait un progrès dans une propension à long terme vers toujours plus d'analytisme et d'abstraction :

Suivant une tendance générale des langues indo-européennes, qu'elles ont menée à son terme extrême, les langues romanes ont ruiné le système de la flexion casuelle. Un seul groupe a gardé jusqu'au moyen âge un reste de déclinaison sous la forme de l'opposition d'un cas sujet et d'un cas régime : le groupe gallo-roman. C'est celui où, de par les habitudes d'employer des parlers germaniques où la flexion casuelle était simplifiée, mais où le sujet s'opposait nettement à diverses formes de cas régimes, des sujets parlants gardaient le sens de l'emploi des cas. La concordance est d'autant plus saisissante que la tendance à ruiner le système des cas persistait, et que, au bout de peu de siècles, une fois l'influence germanique éliminée, elle a abouti : au XIII ${ }^{\mathrm{e}}$ siècle, l'opposition du cas sujet et du cas régime n'était sans doute plus qu'une tradition littéraire. (LHLG 2, 1931a, 96)

Au nombre des traits linguistiques liés au bilinguisme romano-germanique

(...) par tous les individus qui à la fois approchaient des chefs et conservaient une culture intellectuelle, la lingua romana et un parler germanique ont été employés suivant les cas. Donc, dans l'esprit de ces individus, la forme latine et la forme germanique s'équivalaient souvent, et ils pouvaient être amenés à parler latin avec des tours germaniques ou germanique avec des tours latins. ( $L H L G 2,1932 \mathrm{a}, 106)$

Dans cette rubrique, Meillet énumère l'inversion du sujet dans la proposition interrogative, la spécialisation de « on » $(<$ homo $)$ comme un équivalent du « Man » allemand et de « rien » $(<$ rem $)$ en face de « nicht» $(<n i$ wiht, « pas une chose $»)$.

Pour une image de la situation actuelle, il donne en exemple dans les réalisations orales du français par les Provençaux la perte de l'opposition de nombre du déterminant possessif (ils viennent de marier son (vs leur) enfant) et la prononciation des voyelles :

Mais si un Français du Midi parle le français normal fondé sur l'usage de l'Ile-deFrance, il a tendance à se servir par exemple des voyelles de son parler provençal ou gascon. De là résulte que dans le Midi de la France, le français est prononcé par beaucoup de gens avec des voyelles tout autres que celles qui sont normales dans la région parisienne ou, d'une manière générale, dans la France du Nord. Par exemple les voyelles nasales des méridionaux n'ont pas le caractère de pureté qu'ont les voyelles nasales normales du français. (LHLG 2, 1932a, 108)

Les effets du bilinguisme sur la langue française retiennent l'attention. La conception traditionnelle qui voyait dans la présence d'éléments anciens (substrat) ou étrangers (superstrat) la cause d'une déformation de l'organisation phonologique, lexicale et morphosyntaxique d'une langue est réinterprétée par Meillet comme un infléchissement de la tendance multiséculaire qu'exprime au long de son histoire une communauté de locuteurs (le celtisme dans le parler roman), ou comme une modification du rythme suivi par le progrès des langues.

Mais, alors qu'on s'attendrait à lire ces études dans l'Année sociologique de Durkheim, elles sont exposées devant l'auditoire constitué par les classes de l'Institut et livrées aux lecteurs du Journal de Psychologie. Faut-il conjecturer quelque critique du chauvinisme linguistique devant une réunion des académies où siège ce qui se trouve de plus réactionnaire en matière de langue ? Est-ce une contribution critique à la psychologie qui, lorsqu'elle n'oublie pas la langue, élude qu'elles peuvent être plusieurs chez une même personne ? Quoi qu'il en soit, sur une question éminemment sociologique, priorité est donnée à la structure de la langue et à la conscience des "sujets parlants " aux dépens des interactions sociales, comme si une certaine distance était prise avec les méthodes de l'école durkheimienne. 


\subsection{Grammaticalisation et progrès des langues}

Pour Meillet, il y a donc un finalisme dans la diachronie et le français en représente un cas de figure emblématique. Réitérant les propos de sa leçon inaugurale au Collège de France, il affirme :

Le principe sur lequel on voudrait attirer l'attention, c'est que le développement roman continue le développement qui conduit de l'indo-européen au latin. Il n'y a pas eu deux développements successifs, mais un développement continu, tantôt lent et tantôt rapide, qui va de l'indo-européen aux parlers romans actuels. (LHLG 2, 1925 , 114)

Les traits que Meillet identifie comme constitutifs du progrès des langues (encore qu'il évite soigneusement ce mot auquel il préfère « développement ») sont, comme il a été dit, l'analytisme et l'abstraction en plus de la polysémie dont Bréal avait souligné l'importance ( $L H L G$ 1, 1906b, 244). A la suite d'un exposé devant la Société de Psychologie, il répond à une question de J. Vendryes :

Je crois que dans le passage du type indo-européen à une structure française par exemple, il y a réellement une différence d'abstraction : la tendance universelle à éliminer la flexion casuelle n'est pas un accident. D'ailleurs, ce qui intéresse le linguiste, c'est moins la réalité que la forme sous laquelle les choses sont présentées. Or le linguiste se trouve irrécusablement en face d'une structure inégalement abstraite. (LHLG 2, 1923, 16)

Le remaniement de la morphologie, avec le transfert des fonctions casuelles vers des unités autonomes (déterminants, prépositions) et un ordre fixe des mots, rejoint le concept de " grammaticalisation » qu'il avait proposé en 1912 et qu'il a repris dans son article de 1915. Il avait pris pour exemple la disparition du prétérit :

Les deux types de prétérits, le type simple et le type à participe et auxiliaire, peuvent se maintenir indéfiniment côté à côte, avec leur différence de sens initiale. Mais la forme simple qui est ancienne et qui, malgré de nombreuses innovations analogiques, ne parvient jamais à n'avoir qu'une caractéristique unique pour tous les verbes, qui surtout a des aspects très divers dans les verbes forts, et qui enfin a souvent une flexion très singulière (ainsi en français, nous aimâmes, vous aimâtes), se trouve, au point de vue proprement morphologique, en infériorité décisive par rapport à la forme composée qui a pour tous les verbes une seule et même structure. ( $L H L G$ 1, 1909, 155)

Le trait essentiel qui contribue à ce changement, que Meillet mentionne à plusieurs reprises, c'est une autonomisation des unités lexicales par rapport au radical dont elles dérivent, qui est l'un des traits qui distingue, traditionnellement, les langues indoeuropéennes et les langues afro-asiatiques :

En réalité, les altérations violentes que la phonétique a fait subir aux formes sur le terrain gallo-roman, surtout dans le Nord, ont miné le sentiment d'un élément radical. Les mots qui, par eux-mêmes, étaient le mieux fait pour maintenir le sentiment d'un radical étaient les verbes radicaux. Ils ont été si disloqués par le traitement qu'ils ont subi qu'il n'y est, en général, rien resté où l'on ait pu apercevoir un radical. Soit lego. Les formes françaises sont de l'un des types : je lis - nous lisons - je lirai - lu. Le substantif lectionem a abouti à leçon. Le seul élément commun à tous ces mots est $l$ qui ne peut passer pour un radical. Dès lors, rien ne s'opposait à l'adoption de mots venus de la langue écrite : lecture, lecteur. (LHLG 2, 1930,124)

De cette action corrosive du changement phonétique sur la morphologie dérivationnelle, Meillet tire une conclusion à l'avantage de l'état moderne :

Le vocabulaire y gagne en précision : une formation de mots ne peut orienter sur le sens que d'une manière vague. Le vocabulaire est le domaine des faits particuliers ; c'est donc un mérite du français que d'opérer avec des mots dont le sens est fixé d'une manière aussi individuelle et que défigurent le moins possible des reflets jetés par d'autres mots. ( $L H L G ~ 2,1930,127$ )

D'où il conclut : «L'isolement est l'état normal du mot français. En règle générale, il faut se reporter au latin pour comprendre les rapports que soutiennent entre eux les mots français ». ( $L H L G ~ 2,1928,139)$. A l'intersection de l'isolement et de la grammaticalisation, à partir de deux exemples choisis comme prototypiques « il est vache » et « j'ai très faim » (1931b), Meillet voit dans des constructions sans déterminant une translation du nom vers l'adjectif avec être et vers l'adverbe avec avoir. Faisant référence au « génie du français », il voit dans ces évolutions « (...) un ensemble de faits dont il importerait d'étudier le développement dans le passé, et de décrire l'extension dans le parler d'aujourd'hui. Car il s'y manifeste une tendance profonde de la langue française. » (LHLG 2, 1931b, 137) 
Dans l'article de 1906c, Meillet avait donné un autre exemple de cette séparation interne aux familles de mots dans la disjonction entre les noms (populaires) et les adjectifs savants qui ne permettent pas de déduire oculaire de oil par exemple. Il opposait une opération raisonnée d'enrichissement du vocabulaire aux créoles dont les conditions d'apparition ont abouti à des états de langue " privés de presque toute leur grammaire, affaiblis dans leur prononciation, réduits à un petit vocabulaire » $(L H L G 2,1924,68)$, une accélération des changements qui contraste avec la résistance à l'innovation des « grandes langues commune de civilisation » $(L H L G 1,1911,121)$.

\section{Conclusion}

Meillet a dû accepter le partage universitaire entre romanistes et indo-européanistes, entre spécialistes du français et des autres langues de la famille, tout en remontant dans la reconstitution de l'histoire de la langue jusqu'au latin (1906a ; 1928a ; 1932a). Sa contribution sur le français s'est orientée dans deux directions qui formulaient l'une et l'autre une critique des jugements reçus.

D'un côté, Meillet a relu le passé du français en prenant en compte les conditions particulières de son élaboration, dominée par l'activité des cercles lettrés, et de sa diffusion qui s'est opérée pacifiquement à partir des clases dominantes urbaines, sans mouvement migratoire significatif. Au-delà, il a élargi l'observation de ses transformations à l'échelle de l'Europe, refusant de contenir l'observation à une seule langue, et même à la seule famille romane. Alors que les rivalités nationales exacerbées ont abouti à la Première Guerre Mondiale et que le triomphe du fascisme et du nazisme laissaient appréhender un nouveau conflit, Meillet prône un rapprochement européen dont les intellectuels prouveraient la consistance et la faisabilité.

De l'autre, il a cherché à reconstituer un " développement » qui prenne en compte, en plus de changements finalisés et attribués au génie des langues, tels qu'ils se lisent en particulier dans la grammaticalisation, les effets du contact des langues, ouvrant l'explication du changement à l'action du bilinguisme à l'échelle de l'individu et de la société. A ce titre, l'opposition entre un français corseté dans sa tradition littéraire élitiste et la créologenèse déterminerait l'espace dans lequel s'est déployée l'évolution du français parlé dont tel tour, tel mot (par exemple jument) livrent un aperçu.

\section{Références bibliographiques}

Meillet, A.

(1906a). De quelques innovations de la déclinaison latine, Paris : Klincksieck, (1906b). L'état actuel des études de linguistique générale. Revue des Idées, 15 avril. (1906c). Comment les mots changent de sens. L'Année sociologique IX, 1-38.

(1909). Sur la disparition des formes simples du prétérit. Germanisch-Romanische Monatsschrift,

521-526. [+ note ajoutée en 1920 à la republication dans $L H L G 1$

(1911). Différenciation et unification dans les langues. Scientia IX, 402-449.

(1912). L'évolution des formes grammaticales. Scientia XII, 384-400.

(1913). Sur la méthode de la grammaire comparée. Revue de Métaphysique et de Morale, 1-15.

(1915). Le renouvellement des conjonctions. Annuaire de l'Ecole des Hautes Etudes, 5-24.

(1921a). Linguistique historique et linguistique générale [LHLG 1]. Paris : Champion.

(1921b). Jules Gilliéron et l'influence de l'étude des parlers locaux sur le développement du romanisme. $L H L G$ 1, 305-309. Paris : Champion.

(1923). Le caractère concret du mot. Journal de Psychologie, 246-258.

(1924a). (\& Cohen, Marcel) Les Langues du monde. Paris : Champion.

(1924b). Introduction à la classification des langues. Les Langues du monde repris in LHLG 2, 53-69.

Paris : Champion.

(1925). Les interférences entre vocabulaire. Lecture faite à la séance publique des cinq académies le 25 octobre.

(1926). Sur la valeur du mots français jument. Archivio Glottologico Italiano : 147-150.

(1928a). \& Vendryes, J. Traité de grammaire comparée des langues classiques. Paris, Champion, 1928.

(1928b). Les langues dans l'Europe nouvelle ( $2^{\mathrm{e}}$ édition avec une statistique des langues par L. Tesnière). Paris : Payot.

(1928c). Esquisse d'une histoire de la langue latine. Paris : Hachette.

(1929). Le développement des langues. Cahiers de la Nouvelle Journée 15, Continu et discontinu, $119-131$.

(1930). La notion de radical en français. The Romanic Review XXI, 291-295.

(1931a). Sur une période de bilinguisme en France. Comptes rendus de l'Académie des Inscriptions et Belles Lettres, 26-38. 
(1931b). A propos de "Il est vache" et "j'ai très faim". Revue de Philologie française 43, 47-49.

(1932a). Sur les effets des changements de langue. Scientia LI, 91-98.

(1932b). Sur l'étymologie du français, Préface au Dictionnaire étymologique de la langue française

d'O. Bloch et W. von Wartburg, repris dans $L H L G$ 2, 138-151.

(1933). Sur le bilinguisme. Journal de Psychologie, 167-171.

(1936) Linguistique historique et linguistique générale 2. Paris : Klincksieck.

Bergounioux, G. (1990). L'enseignement de la linguistique et de la philologie en France au XIX ${ }^{\mathrm{e}}$ siècle (1845-1897). Archives et Documents de la SHESL, $2^{e}$ série, 2.

Bergounioux, G. \& Lamberterie (de), Ch. (2006). Meillet aujourd'hui. Leuven-Paris : Peeters.

Bloch, O. \& Wartburg (von), W. (1932). Dictionnaire étymologique de la langue française. Paris : PUF.

Bopp, F. (1866-1874). Grammaire comparée des langues indo-européennes. Paris : Imprimerie nationale.

Bréal, M. (1897). Essai de sémantique. Paris : Hachette.

Charle, C. (1985). Les Professeurs de la Faculté des Lettres de Paris, dictionnaire biographique (18091908). Paris : CNRS-INRP.

Diez, F. (1874-1876). Grammaire des langues romanes. Paris : Vieweg.

François, J. (2017) Le siècle d'or de la linguistique en Allemagne. De Humboldt à Meyer-Lübke. Limoges : Lambert-Lucas.

Gauchat, L. (1905). L'unité phonétique dans le patois d'une commune. Aus romanischen Sprachen und Literaturen. Festschrift Heinrich Morf. Halle : Niemeyer, 175-232.

Gilliéron, J. \& Mongin, J. (1905). Scier dans la Gaule romane. Paris : Champion.

Herder, F. (1834). Idées sur la philosophie de l'histoire de l'humanité. Paris : F. G. Levrault.

Prost, A. (1968). Histoire de l'enseignement en France 1800-1967. Paris : A. Colin.

Renan, E. (1871). La Réforme intellectuelle et morale. Paris : Michel Lévy.

Rousselot, P.-J. (1891). Les modifications phonétiques du langage étudiées dans le patois d'une famille de Cellefrouin. Paris : Welter.

Schlegel, F. (1837). Essai sur la langue et la philosophie des Indiens. Paris : Parent-Desbarres.

Schuchardt, H. (2011). Textes théoriques et de réflexion (1885-1925). Limoges : Lambert-Lucas.

Swiggers, P. (1988). Le problème du changement linguistique dans l'œuvre d'Antoine Meillet. Histoire Epistémologie Langage 10-2, 155-166. 